\title{
Active Relationships for Controlling the Propagation of Information and Activities in Databases
}

\author{
Friedrich Kemper, Wolfgang Wilkes, Gunter Schlageter \\ University of Hagen, Department of Computer Science, \\ Feithstr. 140, D-58084 Hagen \\ Germany
}

\begin{abstract}
In many complex applications (design, manufacturing, workflow management, etc.) information about activities on specific objects affect related objects and result in subsequent activities on distant objects. The way in which objects are influenced is dependent on the operational semantics of the relationship. To capture these semantics, we introduce the concept of "active relationships" which contain a description of the activity propagation as an integral part of the relationship type definition. Active relationships allow to separate the specification of local activities and their global consequences and to solve typical problems concerning consistency control, configuration management, resource activation, etc. The paper discusses the features of active relationships and their integration into an object-oriented environment by means of several application examples. In addition, it describes an implementation of active relationships based on an object-oriented database and the use of this implementation for coupling two subsystems to an integrated system.
\end{abstract}

\section{Introduction}

Many application areas, as e.g. design, manufacturing, workflow management, etc., are characterised by their complex object structures: The objects are highly interrelated by different types of relationships with other objects. The relationships are more than simple object pointers, they often bear very specific semantics. Unfortunately, in normal object-oriented systems only semantic-less references can be used to model relationships. As a result, the semantics of relationships has to be coded either into object methods or even into the application programs.

This problem has been recognised by several authors, and various models have been proposed to overcome the problem [BaKi85], [FoTa90, [NgRi91]. But most of these models only introduce pre-defined relationship types which cover some situations.

This research was supported by the ESPRIT project 7364, "JESSI-COMMONFRAME". 
They do not provide a mechanism which enables the user to define new relationship types with application specific semantics.

In this paper we focus on the operational semantics of relationships, namely the propagation of activities and information. In several applications, objects are influenced by related objects: As a consequence of an event on an object, activities are invoked on related objects. The way in which activities are propagated is not a property of a single object, but it is a property of the relationship between the objects.

Several applications can be modelled more easily using this view of objects and their relationships:

- Configuration management: Design objects are organised by many different relationships: They are composed of other design objects (composition hierarchy), they exist in several versions, and they are represented in different, interrelated representations (e.g. schematic and netlist representation of an electronic circuit). The combination of these relationships require complex activity propagations. Some examples: (1) If a component of a composite design object becomes invalid, the composite design object itself has to become invalid. (2) The creation of a version of a component can enforce the automatic creation of a new version of the composite design object. (3) The update of a schematic representation can result in an automatic re-extraction of the corresponding netlist representation.

- Coupling of subsystems: If subsystems are to be coupled, relationships have to be defined between objects in the different subsystems. Activities in one subsystem are propagated via these relationships and cause subsequent activities in the other subsystem.

- Distributed Systems: If the objects are located at different nodes within a distributed system, the co-operation of distant objects is determined by the semantics of the relationships which connect them.

From these examples, we can draw several requirements:

$\Rightarrow \quad$ It is important to distinguish between the local activities on single objects and their consequences for other objects. The specification of the propagation of activities determines the way in which different objects interact. Thus, the specification of the activity propagation is part of the description of overall processes and should not be mixed with the specification of local operations.

$\Rightarrow \quad$ The interaction of objects often needs to be adapted to the needs of a specific environment. Even within a single configuration management system, different departments like to add their specific interaction rules to the general enterprisewide rules. For instance, whereas in general a composite object is set to invalid after one of its components has become invalid, within a design department it might be sensible to invoke a search routine which automatically looks for a similar (valid) component.

$\Rightarrow$ Relationships tend to bear similar semantics. For instance, a version relationship may exist between various object types. To avoid replications of similar 
relationship definitions we need the ability to define generic relationships which can be materialised to concrete relationship types between specified object types.

To fulfil these requirements, this paper introduces the mechanism of active relationships (1) to provide relationships as explicit concepts for the modeller, and (2) to specify the propagation of information and the possible invocation of follow-up actions on related objects as an inherent part of the operational semantics of relationships. Relationship categories allow the definition of generic relationships, and rule environments support a controlled adaptation of the defined activity propagation for specific users and user groups.

The remainder of this paper is organised as follows: In the following section, the technical description of the concept of active relationships is presented. Section 3 describes the implementation of the active relationships in the context of a CADFramework's database and the use of active relationships for the integration of different subsystems. Related approaches are discussed in section 4.

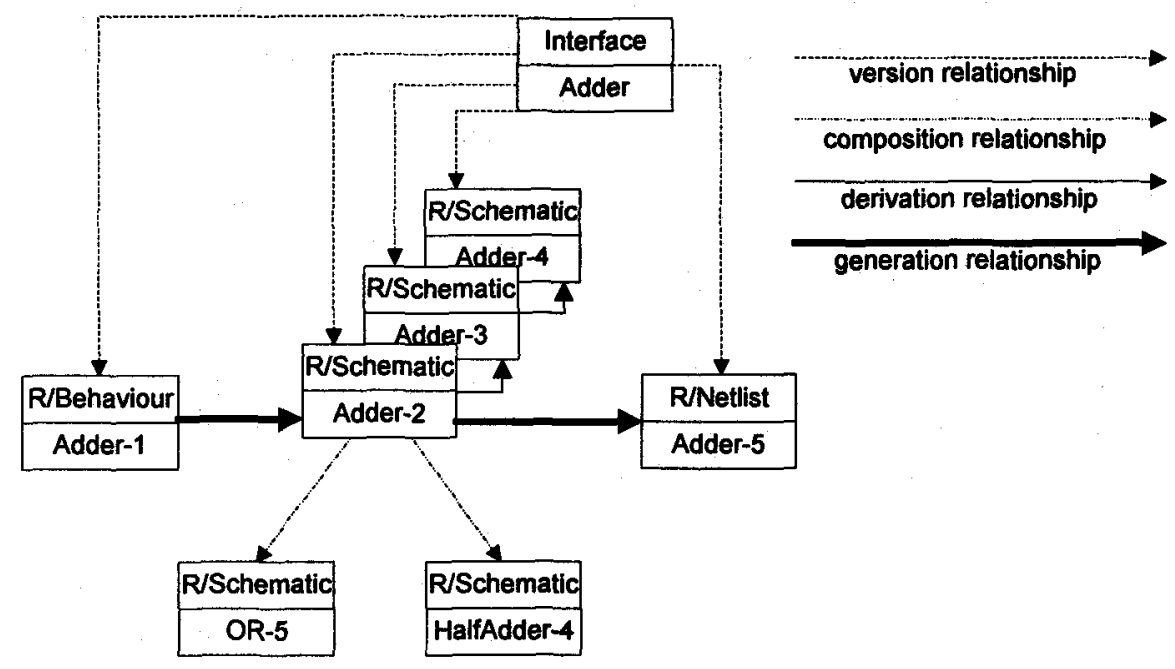

Figure 1: Typical relationships of a CAD application

\section{Active Relationships}

\subsection{Example}

We start the description of active relationships with an example from the area of VLSI design which will be used throughout this section. During the design of an Adder several representations are created which are connected to each other by various relationships (figure 1). The consistency of a single object in this structure is 
determined to a high degree by the objects to which it is related. For example, the schematic representation Adder- 2 can only be as valid as its components are, i.e. if a component is invalidated, the same is true for the composite object. Similarly, Adder2 is dependent on the behavioural representation Adder-1 from which it was generated (e.g. manually by a designer), and the netlist representation Adder-5 is dependent on the schematic representation in the same way. Revisions derived from Adder- 2 are influenced by changes of Adder-2 as well. Moreover, if the interface specifications in the interface object Adder change, all representations which are versions of Adder are influenced since then they are based on an outdated specification. Thus, a lot of operations which take place on different objects may affect the consistency of the object Adder-2, and in the same way, operations on Adder-2 may affect other objects.

Figure 2 shows the schema level of our example, i.e. the involved object types and their relationship types.

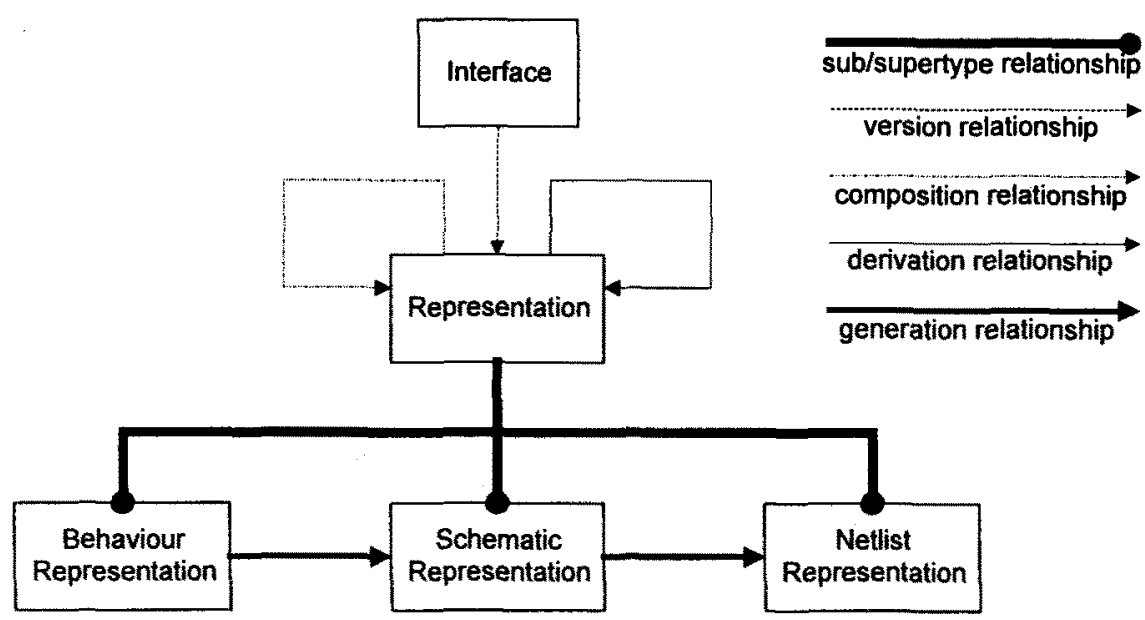

Figure 2: Object types and relationships of the CAD application example

\subsection{Object Types}

The basis for our model is a basic object-oriented data model: An object type defines the attributes which represent properties of the objects of this type, and it defines a set of methods to manipulate these properties. For each attribute, automatically two methods are created:

$\begin{array}{ll}\text { <attribute_name> } & \text { to read the attribute } \\ \text { SET_<attribute_name }> & \text { to modify the attribute }\end{array}$

If an attribute is set-valued, it can be manipulated by three pre-defined methods: 
<attribute_name> $\quad--$ to read the attribute values (this method returns an iterator to run through all elements of the set)

add_to_<attribute_name> $>$ - to add a value to the set

delete_from_<attribute_name> $>$ - to delete a value from the set

Example 1 shows the definitions of the object type SchematicRep and its supertype Representation (only the explicitly defined methods are shown). The syntax is $\mathrm{C}++$ oriented.

\section{Example 1:}

a)

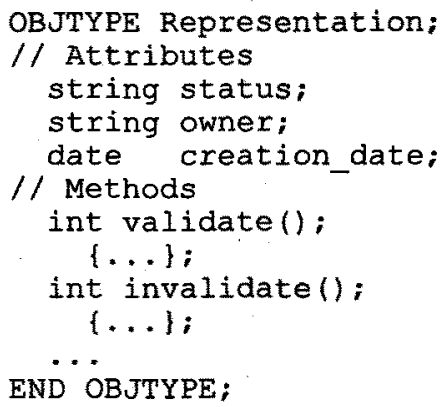

END OBJTYPE;

b)

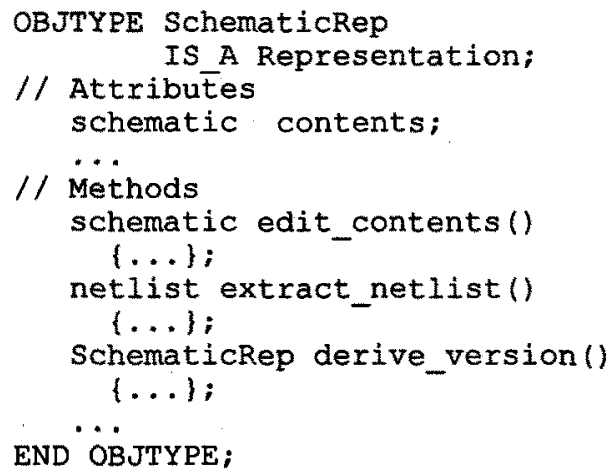

END OBJTYPE;

Of course, the contents attribute defined for SchematicRep objects is a complex structure consisting of several parts (ports, nets, instances, graphical elements, etc.), but within the context of this paper it is sufficient to treat those parts as a whole.

\subsection{Relationship Types}

Relationships between object types are defined explicitly by a relationship construct. In the definition of the relationship type, each of the involved object types gets a specific role name ('[ ]' denote optional parts, "' separates alternative terms):

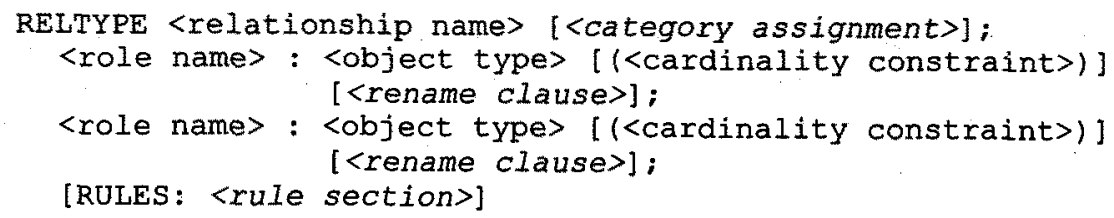


In general, it is also possible to have $n$-ary relationships with $n>2$. But since active relationships are usually binary, transferring an activity from one side to the other one, we only deal with binary relationships in this paper. The rule clause, the category assignment clause, and the rename clause are discussed in the subsequent sections.

The following example shows the definition of the relationship type derivation which is recursively defined on the object type Representation. The relationship type defines a 1:n relationship: Each representation may be derived from at most one predecessor.

\section{Example 2:}

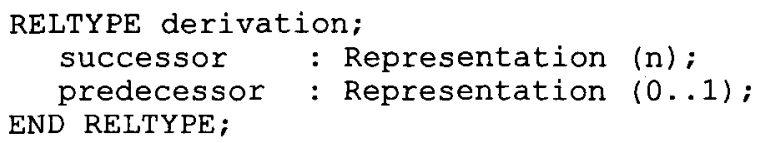

As a result of this relationship definition, the object type Representation "inherits" some additional methods to manipulate the relationships in which it participates. Due to playing the role predecessor in the derivation relationship, Representation is extended by the following operations:

successors: $\quad$ returns an iterator to run through the set of successors add_successor: to relate the Representation object with a new successor delete_successor: to disconnect the Representation object and a given successor

The following methods are added to Representation because it plays the role successor in the derivation relationship:

predecessor:

connect_predecessor:

disconnect predecessor: returns the predecessor of the Representation object to connect the Representation object with a predecessor to disconnect the Representation object from its predecessor

As the example shows, the object type "inherits" set oriented methods if it plays a role which can be connected to more than one object of the other role (as the predecessor role), and it gets methods which work on single objects otherwise (as the successor role). It does not matter from which side a relationship was built (from the successor via the operation connect_predecessor or from the predecessor via the add successor operation), it is always visible form both sides.

The inherited methods are directly applicable, for instance to implement the methods of the object. An example: The method derive_version copies an object of type SchematicRep and relates original and copy by means of the derivation relationship. This is performed by using the "inherited" method add_successor : 
Example 3:

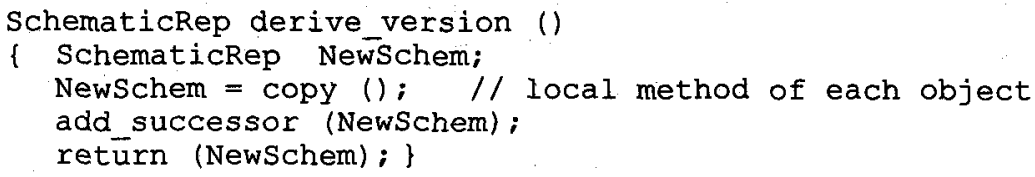

\subsection{Relationship Rules}

The operational semantics of a relationship defines how it propagates changes from one side of the relationship to the other one(s). This propagation behaviour is defined by means of relationship rules, a special kind of ECA rules known from the area of active databases [Daya88], [Buch94]. A relationship rule is defined locally to a relationship. Consequently, it can only recognise events and use methods of those objects which are related by this relationship. Figure 4 shows the structure of a relationship rule definition.

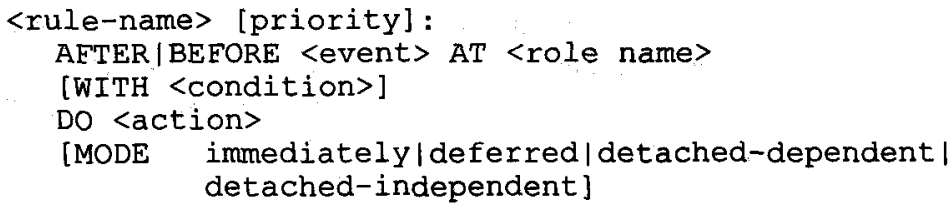

Figure 4: Definition of a relationship rule

A relationship rule fires whenever the given event is raised. If the given condition is evaluated to true, the specified action is executed. The mode part of the rule definition corresponds to the coupling modes commonly used in active databases [Daya88], [Buch94]. It determines whether the rule is executed in (respectively at the end of) the transaction which raised the event ("immediately"/"deferred") or whether the firing of the rule creates a new transaction ("detached"). If this new transaction is "dependent" to the running transaction, it may not start before the termination of the running transaction. The default mode is "immediately".

Example 4 shows a relationship rule associated to the composition relationship. It causes a composite object to become invalid as soon as one of its components is invalidated. The rule is triggered by an invalidation of the status attribute of the component object and invalidates the composite object as well. 


\section{Example 4:}

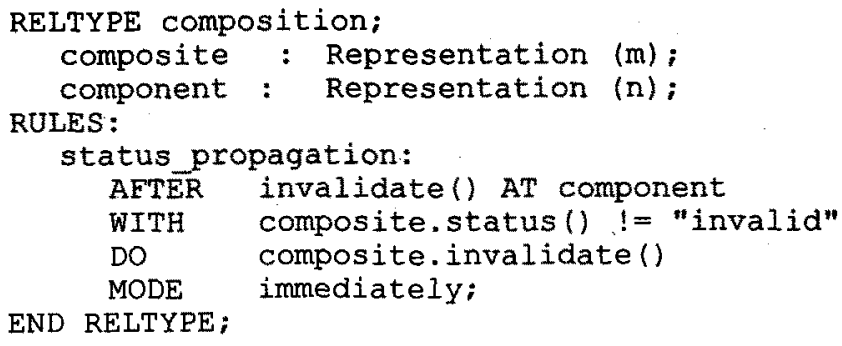

Another example enriches the copy function which was already used in the implementation of the method derive-version. In general, a copy can be performed in three different ways (see [Rumb88]:

1. Copy only the local attributes.

2. Copy relationships as well (i.e. relate the copy to those objects which are related to the original).

3. Copy also those objects which are related to the original (deep copy).

If we consider our example (figure 1 and 2), then it is clear, that our copy has to perform for some relationships like alternative 2, for others like alternative 1: Whereas a copy of a Representation object $\mathbf{R}$ should have the same components as $\mathbf{R}$ and should also be related to the same Interface object as $R$, it cannot be connected to other objects by means of the derivation relationship in the same way as R. Example 5 describes the effect of a copy operation for the components by a relationship rule.

\section{Example 5:}

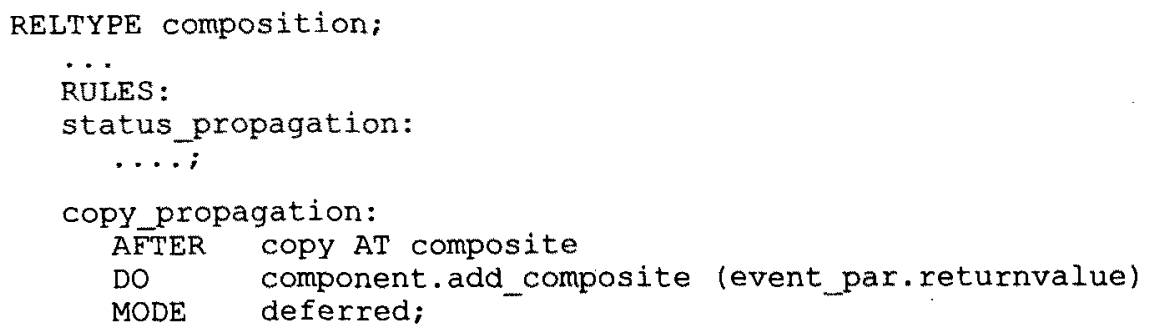

The rule causes each component to be automatically connected to the new copy of its composite object. This copy can be accessed via the parameter event_par.returnvalue (see below). In the following, the single parts of a relationship rule definition will be discussed in more detail.

\section{Events}

Two classes of events are of interest:

- database events: events which can be used to observe the manipulation of data, i.e. creation, deletion, or update of an object.

- method events: events which indicate the invocation or termination of a method. 
In both cases we can distinguish pre- and post-events, i.e. the event may be raised before the update or the execution of a method or after the update or the method execution (indicated by keywords BEFORE or AFTER).

A method event allows to access the actual parameters of the method which raised the event. In case of a post-event, also the return value of the method is provided. The access to parameters and return values is done by event_par.<parameter-name $>$ and event par.returnvalue respectively.

\section{Conditions and Actions}

Conditions control the execution of the rule action. They can be expressed as arbitrary boolean expressions. Only if the condition evaluates to true, the action part is executed. The action part contains a block of statements of the underlying programming language (in which the object methods are defined).

As an example for a relationship rule with a complex action part consider the specific kind of change propagation which has been introduced in [Katz90] (see figure 5): A derivation of a new version of one of the components of a composite object (a) leads to an automatic creation of a new version of the composite object (b). This mechanism works recursively until the root object of the component hierarchy is reached or the scope of the propagation is limited by means of a flag in one of the composite objects.

a)

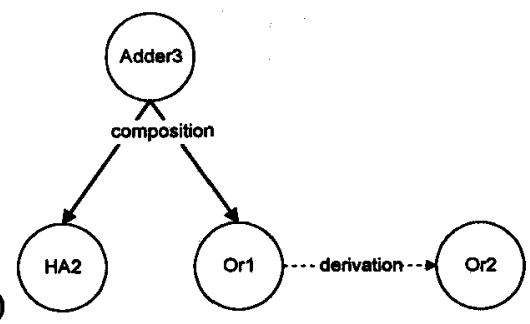

b)

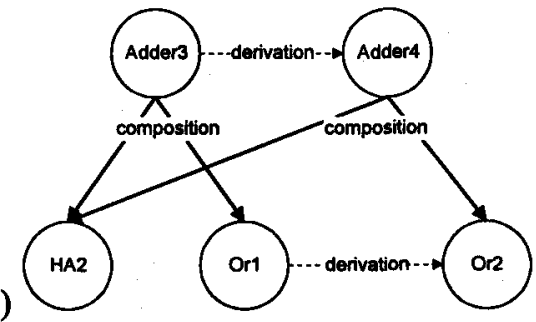

Figure 5: Change propagation according to [Katz90]

This behaviour can be modelled by associating the following relationship rule to the composition relationship:

Example 6:

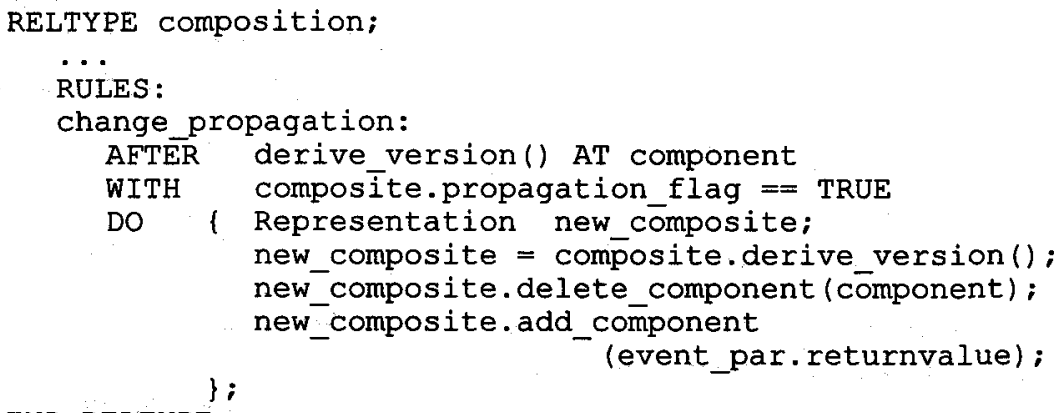




\section{Synchronisation and Termination}

As know from the field of active databases, triggering of activities may cause several problems [Etz93]. One problem is the synchronisation of actions triggered by the same event. A single event may raise more than one relationship rule and may thus lead to the execution of several actions. Those actions can be executed in parallel without any problems if the actions are independent from each other (i.e. if they access different data). But, if dependencies exist between the triggered actions, then appropriate priority numbers which control their execution order must be assigned to the respective rules (see priority field in figure 3 ).

A common problem of all approaches which uses ECA rules is non-termination. In the area of relational databases several models have been developed which allow the detection of (potential) cycles [AWH92], [BCW93], [VoSi93]. Some additional problems arise in the area of object-oriented systems (among other things due to the encapsulation of methods). [Buch94] sketches two possible solution of this problem. In our current implementation we use a simple time-out mechanism.

\subsection{Relationship Categories}

Usually a lot of different relationship types exist in a specific application, but several of them bear a similar semantics. This has been illustrated in figures 1 and 2 , where the same kind of relationship has been used to relate the object types SchematicRep and BehaviourRep as well as NetlistRep and SchematicRep.

To support the reuse of relationship definitions we introduce relationship categories. A relationship category builds a template for the definition of actual relationship types (see also [KrWi95]). A relationship type which is bound to a specific category inherits the roles and rule specifications of its category. The object types of the inherited roles may be specialised to subtypes. In the following example, generation is a relationship category where both roles are of type Representation. This category describes common properties of the relationships between BehaviourRep and SchematicRep and between SchematicRep and NetlistRep respectively.

\section{Example 7:}

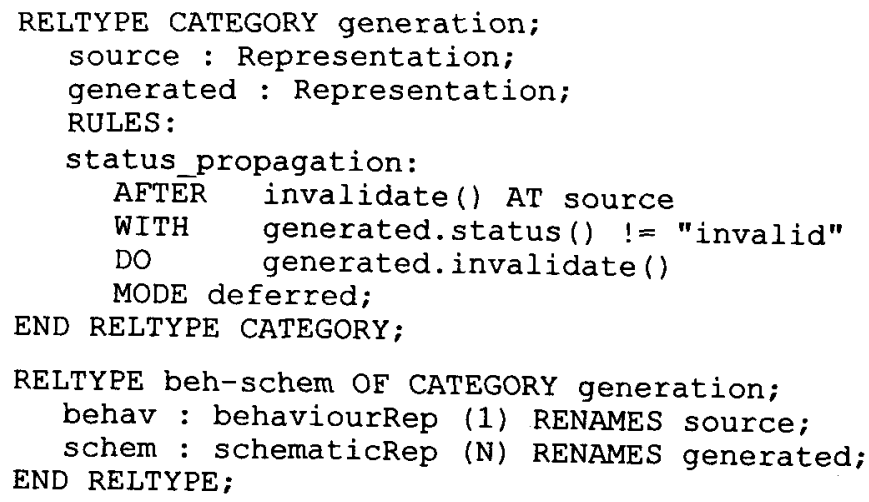




\section{Example 7 (continued):}

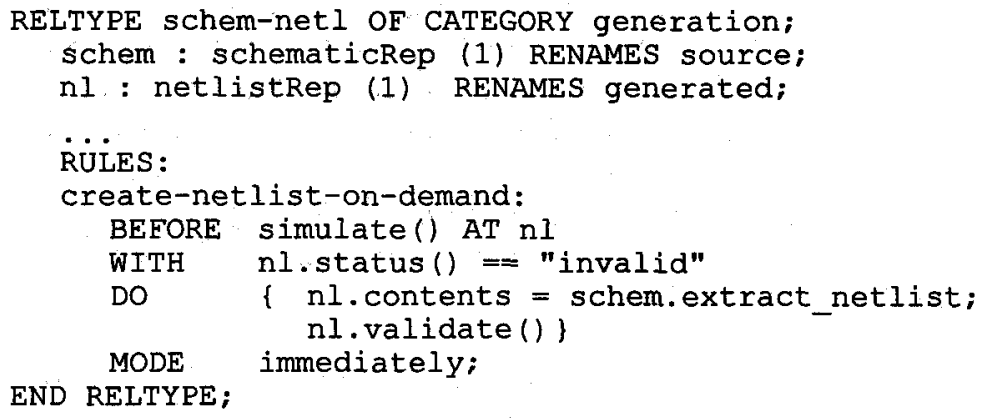

In this example, the category generation defines the rule status_propagation which is inherited by both relationship types of this category. In the relationship schem-netl an additional rule has been defined to extract the actual netlist structure if a netlist simulator is invoked. The rename clause allows to redefine role names which have been inherited from the category.

\subsection{Rule Environments}

The activity propagation defined by active relationships determine to a great deal the way how users can work with the system. But often, even within the same project, different designers will have a different understanding of the desired system behaviour. Some of them would prefer an automatic (re-) creation of objects whenever possible. Others - more experienced ones - would prefer to use only status propagation and notification services. To support a flexible usage of relationship rules, we provide the concept of rule environments to adapt the system behaviour to the needs of (groups of) designers. Rule environments are extensions of the ETM environments defined in [Kotz89].

A rule environment can be seen as a kind of database view. It contains all object and relationship types defined within the database schema and a (possibly empty) set of relationship rules. Each database access happens via a rule environment and only those rules are active which have been attached to the environment. A rule environment is defined by

DEFINE ENVIRONMENT <env_name> [SUB-ENV OF <superenv-name>]

and rules are attached to it by

ATTACH <rule_name> TO <env_name> [IRREVOCABLE] .

Rule environments may be created as sub-environments of other environments (its super-environments). Each environment has at most one super-environment but any number of sub-environments. This results in a hierarchic structure of rule environments, and a single environment inherits the relationship rule attachments of all its predecessors. In addition, further rules can be attached to it. 
Our concept supports two different modes of attachment inheritance which depend on the chosen form of the attach-operation.

- Default inheritance: If the respective rule has been attached to the environment without using the IRREVOCABLE option, then the rule can be detached in one of the sub-environments. A rule of this kind represents a system behaviour which is recommended but not enforced.

- Strict inheritance: A relationship rule which has been attached as IRREVOCABLE must stay active in all sub-environments and cannot be detached there. This allows to enforce a common behaviour within a rule environment and to make it also obligatory for each sub-environment. This feature can be used, for instance, to enforce company or group specific policies.

The combination of these two modes allows to define a common system behaviour which is recommended for all users of the system but can be adapted - within the given limitations - to specific needs of groups of users or even to individual needs of single users. This allows to apply the semantics of relationships in a very flexible way.

\section{Implementation and Sample Application}

The research presented within this paper has been performed in the context of the ESPRIT project JESSI-Common-Frame [JCF92], a collaborative project of many partners, among them Siemens, Siemens Nixdorf Information Systems, Philips and ICL. The result of the project was a CAD framework for the area of micro electronics called JESSI-Common-Framework (JCF) which is now commercially available under the name SIFRAME. JCF provides - based on an object-oriented database mechanisms for data and design management, tool integration, tool communication, etc.

Our implementation of active relationships is based on JCF's underlying active object-oriented database system OMS [FFH+91]. This database provides a simple side effect management which monitors the execution of basic database operations (create_object, delete_object, modify_attribute, create_relationship, ...). OMS supports only event/action rules, i.e. the evaluation of conditions is not supported. Actions can be described by arbitrary user-defined methods.

We have implemented an additional layer on top of the OMS database which allows the management of active relationships [KeWi95], [Emam96]. This layer uses the existing OMS event handling component to detect relevant modifications of data. Each event defined within a relationship rule is mapped onto a corresponding OMS event. If the modification of an object has been recognised, the active relationship layer checks whether a relationship is sensitive to this event. If this is the case the given condition is evaluated by the active relationship layer. Conditions are described by expressions in the Lisp-like language Scheme. If the condition evaluates to true, the corresponding action is executed. 
The system provides much flexibility: Relationship rules may be defined or deleted at runtime, and they may be temporary deactivated. It is also possible to define specific rule environments for different projects.

The implementation of the mechanism of active relationships has actually been used to support the integration of a subsystem into the existing design management component of JCF. In general, the problem of integrating two subsystems can be sketched as follows (see figure 7):

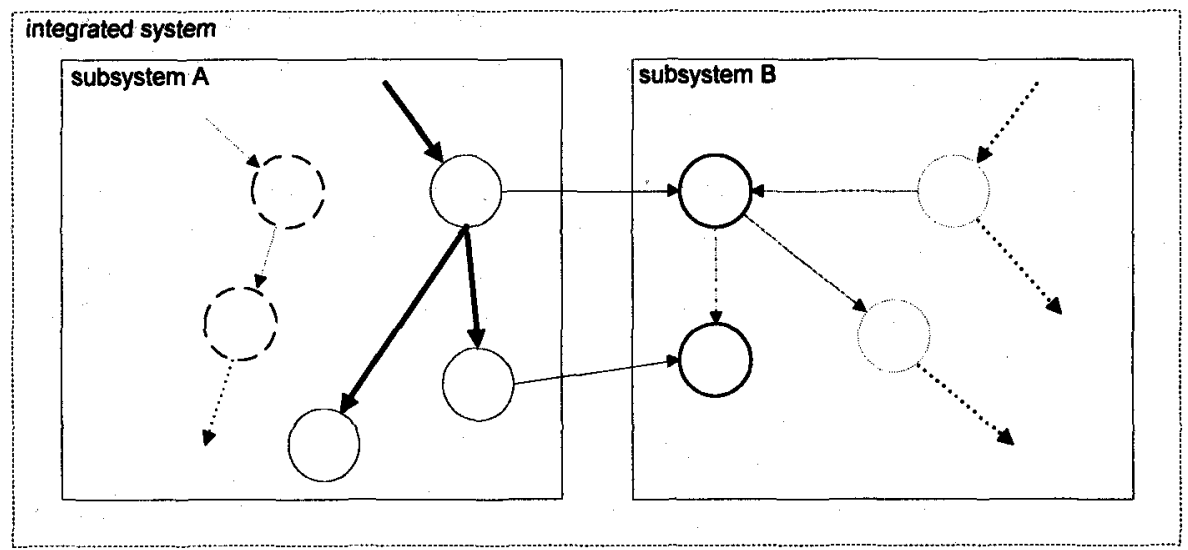

Figure 7: Two subsystems which are related to each other via several relationships

Both subsystems provide inherent functionality which is implemented based on their local object and relationship types and the corresponding methods. To combine the subsystem, some relationships have to be established between objects in different subsystems. Via these relationships, operations in one system influence the objects of the other system. These dependencies can be described and maintained by means of active relationships.

Our extension of the design management component improves the version management of composite design objects. For this purpose we add a so called "configuration tree" to the JCF component hierarchy. Details about the functionality of the configuration tree can be found in [WiKe94]. To integrate our configuration tree to the design management component, we defined relationships between elements of the component hierarchy and the configuration tree. Since it is not possible to change the code of the JCF design management system, we used active relationships to monitor the behaviour of the objects of the design management component, and in case of relevant events, the necessary actions were initiated on related objects in our configuration tree. More details about this coupling can be found in [KWS95], [KeWi95] and [Emam96]. 


\section{Related Approaches}

All ER-based models support the concept of relationships as an explicit modelling mechanism. An advanced example of this type of model is presented in [AGO91], where relationships are modelled as n-ary associations. Properties of associations are specified as constraints (like referential, surjectivity, or constancy constraints). By using various declarations it is possible to maintain the referential constraints and even more complex pre- and postconditions for insert and delete operations.

In [Rumb87] and [Rumb88] an approach is presented which is based on an objectoriented language. Rumbaugh introduces $n$-ary relationships which allow to specify the propagation of operations across relationships via so called propagation attributes which are attached to the relationship types. This mechanism supports only the propagation of the triggering operation but not the invocation of different actions.

More possibilities are provided by the software configuration management system Adele [EsCa94] and the SORAC database management system [DPW93]. Normally, frameworks and configuration management systems contain hard-wired strategies for the resolution of consistency conflicts across relationships (e.g. [Katz90], [JCF92], [Wolf94]). But Adele and SORAC allow to more explicitly express the semantics of a relationship. Actions on objects can be associated to events (changes of related objects), and their execution is controlled by boolean conditions.

However, both systems do not provide any means to describe a common behaviour of several relationships. Moreover, all rules are defined as global rules which are operative for the whole system. Adaptations to the special needs of single users are not supported. A major difference of our approach to the SORAC system and to the other approaches is the way how relationships are embedded into an underlying object-oriented system and how objects present themselves to applications. As opposed to the SORAC system which provides specific object structures to capture the relationships which have to be known by the application, in our model the application can use the usual methods for dealing with relationships (like navigation, creation of a new relation or deletion of a relation). The operational semantics of the active relationship is treated as a side effect of operations on the involved objects. Thus, existing applications are not affected by the definition of the activity propagation, they can treat active relationships in the usual object-oriented way. With respect to this aspect, our approach is very similar to ADAM [Diaz95].

\section{Conclusion}

In this paper, active relationships have been introduced as a mechanism to specify the propagation of activities across relationships. The key features of active relationships are the following:

- Relationship types are represented as explicit constructs for the designer of the system. For the applications, the existence of relationships is visible by specific methods which are inherited by the involved object types.

- The operational semantics of a relationship type, i.e. the way in which activities are propagated across the relationships, is described in relationship rules as part of the relationship type definition. 
- Relationship rules can be seen as local ECA rules in the scope of a relationship type, i.e. only the methods defined for the related object types may be used in specifying the condition and the action part of the rule.

- Relationship categories form templates from which actual relationship type definitions can be derived which inherit the specification of the category.

- Rule environments allow the flexible application of relationship rules in different circumstances.

There are several application fields where active relationships can be of help, including

- consistency control in complex relationship structures

- workflow management: description of the activity flow, invoking of automatable activities, notification of people with a specific role about pending activities, etc.

- coupling of subsystems

The concept of active relationships has been implemented on top of an objectoriented database system with active elements. This prototype has been successfully used for the extension of a design management system by coupling an additional module with the existing system via active relationships.

\section{References}

[AGO91] A. Albano, G. Ghelli, R. Orsini: A Relationship Mechanism for a Strongly Typed Object-Oriented Database Programming Language, Proc. 17th Int. Conf. on Very Large Data Bases (VLDB), Barcelona, Spain, September 1991

[AWH92] A. Aiken, J. Widom, J.M. Hellerstein: Behaviour of Database Production Rules: Termination, Confluence, and Observable Determinism, Proc. ACM SIGMOD Int. Conf. on Management of Data, San Diego, USA, June 1992

[BaKi85] D.S. Batory, W. Kim: Modeling Concepts for VLSI CAD Objects, ACM Transactions on Database Systems, Vol.10, No.3, September 1985

[BCW93] E. Baralis, S. Ceri, J. Widom: Better Termination Analysis for Active Databases, in N.W. Paton, M.H. Williams (Eds.): Rules in Database Systems, Workshops in Computing, Springer, September 1993

[Buch94] A.P. Buchmann: Active Object Systems, in A. Dogac. M.T. Özsu, A. Biliris, T. Sellis (Eds.):Advances in Object-Oriented Database Systems, Springer Verlag, 1994

[Daya88] U. Dayal: Active Database Management Systems, Proc. 3rd Int. Conf. on Data and Knowledge Bases, Jerusalem, Israel, Juni 1988

[Diaz95] O. Díaz: The operational semantics of user-defined relationships in object oriented database systems. Data \& Knowledge Engineering, No. 16, 1995

[DPW93] M. Doherty, J. Peckham, V.F. Wolfe: Implementing Relationships and Constraints in an Object-Oriented Database Using a Monitor Construct, in N.W. Paton, M.H. Williams (Eds.): Rules in Database Systems, Workshops in Computing, Springer, September 1993 
[Emam96] T. Emami: Integration of Active Relationships into a CAD Framework to realize a flexible Consistency Control, Diploma thesis, University of Dortmund / University of Hagen, February 1996 (in German)

[EsCa94] J. Estublier, R. Casallas: The Adele Configuration Manager, in W.F. Tichy (Ed.): Configuration Management, Trends in Software, Wiley 1994

[Etzi93] O. Etzion: PARDES - A Data-Driven Oriented Active Database Model, Sigmod Record, Vol. 22, No. 1, March 1993

[FFH+91] W. Fox, J. Friedrich, R. Hopp, T. Kathöfer, A. Meckenstock, D. Nolte, K. Pielstiecker, G. Reitmeyer, R. Rupprecht, M.Schrew: The Architecture of the Object Management System within the Cadlab Framework, in F.J. Rammig, R. Waxman (Ed.): Proc. 2nd Int. IFIP Workshop on Electronic Design Automation Frameworks, North-Holland, 1991

[FoTa90] S. Foo, Y. Takefuji: Databases and Cell Selection Algorithms for VLSI Cell Libaries, IEEE Computer, Vol. 23, No. 2, 1990

[JCF92] B. Steinmüller: The JESSI-COMMON-FRAME Project - A Project Overview, in M. Newman, T. Rhyne (Eds): Electronic Design Automation Frameworks, NorthHolland, 1992

[Katz90] R.H. Katz: Toward a Unified Framework for Version Modeling in Engineering Databases, ACM Computing Surveys, Vol. 22, No.4, December 1990

[KeWi95] F. Kemper, W. Wilkes: Integration of Active Relationships into JCF, Esprit Project 7364, Document-No. JCF/FU-Hagen/015-01/28-Apr-95

[Kotz89] A.M. Kotz: Triggermechanisms in Database Systems, Informatik-Fachberichte 201, Springer, 1989 (in German)

[KrWi95] T. Kretzberg, W. Wilkes: EXPRESS+ and SDAIt: Specification and Automatic Derivation of Higher Level Programming Interfaces. Proc. 5th Int. EXPRESS User Group Conference, Grenoble, October 1995

[KWS95] F. Kemper, W. Wilkes, G. Schlageter: Active Relationships: A Means for Controlled Propagation of Information and Activities in Databases, Informatik Berichte 188, University of Hagen, Germany, June 1995

[NgRi91] G.T. Nguyen, D. Rieu: Representing Design Objects, Artificial Inteligence in Design, Oxford, Butterworth/Heinemann, 1991

[Rumb87] J. Rumbaugh: Relations as Semantic Constructs in an Object-Oriented Language, OOPSLA '87, Special Issue of SIGPLAN Notices, Vol. 22 , No. 12, December 1987

[Rumb88] J. Rumbaugh: Controlling Propagation of Operations using Attributes on Relations, OOPSLA '88, SIGPLAN Notices, Vol. 23, No. 11, November 1988

[VoSi93] L. von der Voort, A. Siebes: Termination and Confluence of Rule Execution, 2nd Int. Conf. on Information and Knowledge Management, November 1993

[WiKe94] W. Wilkes, F. Kemper: Application of Indirect Components for Version Management in Design Databases, International Conference on Data and Knowledge Systems for Manufacturing and Engineering, Hong Kong, May 1994

[Wolf94] P. van der Wolf: CAD FRAMEWORKS: Principles and Architecture, Kluwer Academic Publisher, 1994 